\title{
Macroalgas varadas sobre la superficie de una playa arenosa del sur de Chile: preferencias alimentarias y de hábitat de juveniles y adultos de Orchestoidea tuberculata (Nicolet), (Amphipoda, Talitridae)
}

\author{
Stranded algal wracks on a sandy beach of south central Chile: feeding and habitat \\ preferences of juveniles and adults of Orchestoidea tuberculata (Nicolet), \\ (Amphipoda, Talitridae)
}

CRISTIAN DUARTE*, EDUARDO JARAMILLO \& HERALDO CONTRERAS

Instituto de Zoología, Universidad Austral de Chile, Valdivia, Chile;

* e-mail para correspondencia: cduarte@uach.cl

\begin{abstract}
RESUMEN
El anfípodo Orchestoidea tuberculata, es el carroñero numéricamente dominante en los niveles superiores del intermareal de las playas arenosas del centro sur de Chile. La principal fuente de alimento para juveniles y adultos de este anfípodo son las macroalgas varadas en la playa. Con el objetivo de analizar una eventual partición de ese recurso alimentario por parte de esos juveniles y adultos, se realizaron experimentos tendientes a evaluar preferencias alimentarias sobre las tres macroalgas varadas más comunes de estas playas: Durvillaea antarctica, Macrocystis pyrifera y Lessonia nigrescens. Los juveniles prefirieron consumir $L$. nigrescens, mientras que los adultos mostraron una preferencia significativa por D. antarctica. Similar a lo registrado en condiciones de laboratorio, los resultados de estudios de terreno muestran que los juveniles de $O$. tuberculata no presentaron preferencias significativas entre $D$. antarctica y $M$. pyrifera, en tanto que los adultos prefirieron los restos de D. antarctica por sobre los de M. pyrifera. Esto sugiere que para los adultos, $D$. antarctica es una fuente importante de alimento y también de hábitat. Debido a que L. nigrescens no pudo ser incluida en los experimentos de terreno, la posibilidad de que los juveniles también prefieren a esta macroalga en esas condiciones permanece como una pregunta abierta. Aunque no se conocen las razones para explicar las diferencias en las preferencias alimentarias de juveniles y adultos, pueden proponerse dos posibilidades: cambios ontogénicos en la morfología de las estructuras bucales utilizadas para alimentación y/ o cambios ontogénicos en los requerimientos nutricionales. Sin embargo, el hecho de que los juveniles prefirieron consumir en condiciones de laboratorio, la macroalga con el menor contenido nutricional $(L$. nigrescens), sugiere otro tipo de explicación a las diferencias observadas. Esta dice relación con el hecho de que al preferir ítemes alimentarios diferentes, los juveniles pueden evitar el canibalismo de los adultos, interacción biológica previamente demostrada para O. tuberculata. La diferencia notoria en los patrones de preferencia de juveniles y adultos de este anfípodo demuestra la importancia de considerar la variabilidad intraespecífica en este tipo de estudios.
\end{abstract}

Palabras clave: playas arenosas expuestas, macroalgas varadas, preferencias alimentarias, anfípodos, centro sur de Chile.

\begin{abstract}
The amphipod Orchestoidea tuberculata, is the numerically dominant scavenger in the upper intertidal levels of sandy beaches from south central Chile. The main food source for juveniles and adults of this amphipod are the macroalgae stranded on the beach. Experiments of food preferences were carried out to analyze the eventual partition on the most common macroalgae on that beaches (Durvillaea antarctica, Macrocystis pyrifera y Lessonia nigrescens) by .juveniles and adults of this amphipod. While juveniles preferred to consume L. nigrescens, adults showed a significant preference for $D$. antarctica. Similar to the laboratory results, that of field studies shows that juveniles of $O$. tubercula ta did not show significant preferences between $D$. antarctica and $M$. pyrifera, while adults preferred to consume $D$. antarctica instead of $M$. pyrifera. That suggest that D. antarctica is an important source of food (and habitat), for adult amphipods. Due to the fact that $L$. nigrescens could not be included in the field experiments, the possibility that juveniles also prefer this macroalgae in those conditions remains as an open question. Although the reasons underlying the differences in the food preferences of juveniles and adults are unknown, two possibilities can be proposed:
\end{abstract}


ontogenetic changes in the morphology of mouth structures used for feeding and/or ontogenetic changes in nutritional requirements. However, the fact that juveniles preferred to consume the macroalgae with the lowest nutritional content (L. nigrescens) among the three species offered as food, suggest another explanation to the observed differences. This refers to the fact, that by preferring different food ítems, juveniles can avoid the cannibalism of adults, biological interaction previously demonstrated for $O$. tuberculata.

Key words: exposed sandy beaches, stranded macroalgae, food preferences, amphipods, south central Chile.

\section{INTRODUCCIÓN}

Las playas arenosas expuestas se caracterizan por la ausencia de macroalgas adheridas al sustrato; por lo tanto, la mayor parte de la macroinfauna que habita en estos hábitat costeros está representada por especies carroñeras o suspensívoras, las primeras dependientes de subsidios tróficos provenientes de otros sistemas costeros (Griffiths \& StentonDozey 1981, McLachlan et al. 1981, Griffiths et al. 1983). Por lo tanto, el subsidio de alimentos y nutrientes desde otros sistemas, puede tener importantes efectos sobre la macroinfauna de las playas arenosas. Una de las principales fuentes de materia orgánica en este tipo de ambientes, proviene del arribo de macroalgas (e.g., Griffiths et al. 1983, Inglis 1989, Dugan et al. 2003, Jaramillo et al. 2006). Estas macrófitas pueden influir sobre la estructura comunitaria y poblacional de la macroinfauna, sirviendo como alimento y/o refugio (e.g., Duarte 1974, Griffiths et al. 1983, Pennings et al. 2000). Por ejemplo, Griffiths et al. (1983), mostraron que el $71 \%$ de las algas depositadas en un año, sobre la zona intermareal de una playa de Sudáfrica, fue consumida por la macrofauna supralitoral, mayoritariamente representada por anfípodos talítridos.

Diversos factores físicos y biológicos pueden influir sobre el desprendimiento de las macroalgas desde los fondos rocosos (Duarte 1974, Dayton et al. 1992, Rodríguez 1999, Pennings et al. 2000). Entre los factores abióticos, las tormentas son la principal causa de ese desprendimiento (Tegner \& Dayton 1987). A su vez, el daño estructural producido en las macroalgas por invertebrados epibénticos, es el principal factor biológico que influye en esos desprendimientos (Dayton et al. 1984). Las frondas a la deriva o aquellas depositadas en ambientes intermareales costeros, pueden encontrarse en estado fresco o en proceso de descomposición, lo cual depende del tiempo transcurrido después del desprendimiento del sustrato rocoso. Inglis (1989) estudió el proceso de degradación de la macroalga Macrocystis pyrifera (Linnaeus) C. Agardh en una playa arenosa de la costa de Sudáfrica, y encontró que la acción microbiana fue el principal agente involucrado en la descomposición de los restos de esa macrofita en la zona supralitoral. La deshidratación es otro de los mecanismos que puede influir significativamente sobre las características de los restos de macroalgas. Por ejemplo, Griffiths \& Stenton-Dozey (1981) registraron que en una playa de Sudáfrica las macroalgas varadas en la zona intermareal llegaron a perder aproximadamente un $50 \%$ de agua durante los primeros diez días después de quedar depositadas en la zona intermareal.

Existe una amplia variedad de rasgos de las macrófitas que pueden influir sobre los patrones de preferencia de los consumidores herbívoros, tales como tipo y concentración de metabolitos secundarios, calidad nutricional, dureza y pH (Steneck \& Watling 1982, Pennings et al. 1998, McClintock \& Baker 2001). Además, esos herbívoros pueden responder de manera diferente a un rasgo en particular (Pavia et al. 1997, Zimmer 1999). Por ejemplo, Pennings et al. (2000) estudiaron la conducta de alimentación del anfípodo Megalorchestia californiana (Brandt) sobre una playa arenosa de la costa de California (USA) y encontraron que esta especie tendió a preferir los restos de macroalgas en descomposición por sobre aquellas en estado fresco. Esto fue explicado por la mayor concentración de los contenidos orgánicos y minerales de las primeras. Por otro lado, en la costa norte de Francia, Adin \& Riera (2003) encontraron que el anfípodo Talitrus saltator (Montagu) utilizó preferentemente algas pardas por sobre algas verdes y rojas como fuente de alimento, a pesar de que las primeras son generalmente consideradas de menor valor nutritivo. Sin 
embargo, las preferencias alimentarias no solo están en función de la calidad nutricional del alimento, ya que factores tales como competencia y riesgo de depredación pueden restringir la elección de una dieta óptima (e.g., Duffy \& Hay 1991, Bednekoff 1996, Jormalainen et al. 2001). Por ejemplo, Jormalainen et al. (2001), estudiaron los patrones de alimentación del isópodo Idotea baltica (Pallas) en la costa de Finlandia y encontraron que la elección de la dieta de las hembras estuvo subordinada al riesgo de depredación.

A pesar de las evidencias que sugieren que el subsidio trófico de las macroalgas varadas tiene un rol importante en las cadenas alimentarias de playas arenosas expuestas, la utilización de este tipo de detritus por parte de la macroinfauna ha sido escasamente estudiada (Griffiths \& StentonDozey 1981, Griffiths et al. 1983, Pennings et al. 2000, Adin \& Riera 2003). Los estudios realizados hasta la fecha se han enfocado principalmente a dar cuenta de las diferencias a nivel interespecífico en los patrones de alimentación de la macroinfauna (e.g., Griffiths \& Stenton-Dozey 1981) o entre distintos ítemes alimentarios para una misma especie (e.g., Adin \& Riera 2003). Sin embargo, algunos estudios sugieren que también pueden ocurrir diferencias en los patrones de alimentación a nivel intraespecífico. Por ejemplo, Scapini et al. (1992) mencionan que los requerimientos energéticos de los juveniles del anfípodo Talitrus saltator son mayores que los de sus adultos conespecíficos, lo que explicaría el mayor tiempo ocupado por los primeros en las actividades de forrajeo sobre la superficie de la playa. Similarmente, Muir (1977) registró en una playa de la costa de Sudáfrica, que la tasa de consumo de los juveniles de Talorchestia capensis (Dana) fue más del doble que la de los adultos.

El anfípodo Orchestoidea tuberculata (Nicolet), es la especie carroñera más común en los niveles superiores de la zona intermareal de las playas arenosas expuestas de la costa centro sur de Chile (ca. $35-40^{\circ} \mathrm{S}$ ) (Jaramillo et al. 2006), representando las macroalgas varadas su principal recurso alimentario. Duarte (1974) estimó para la playa de Mehuín (ca. $39^{\circ} \mathrm{S}$ ) que este anfípodo (1.000 individuos $\mathrm{m}^{-2}$ ) podía consumir hasta $21,8 \mathrm{~kg} \mathrm{~m}^{-2}$ (peso fresco) de algas. De ahí que las abundancias poblacionales bajo cúmulos de macroalgas varadas en playas arenosas del centro sur de Chile, sean significativamente más altas que en áreas de esas playas carentes de los mismos (Jaramillo et al. 2006). Tales abundancias han sido evaluadas a partir de muestreos realizados durante el día, cuando la mayor parte de la población no muestra actividad locomotriz sobre la superficie de la playa (Jaramillo et al. 1980, 2003). Ya que estos crustáceos poseen desarrollo directo, juveniles y adultos coexisten sobre la superficie de la playa durante sus períodos de actividad locomotriz, aun cuando los máximos en esa actividad ocurren a diferentes horas (Jaramillo et al. 1980, 2003).

En este estudio se analizan las preferencias alimentarias y de hábitat de juveniles y adultos de O. tuberculata en condiciones de laboratorio, sobre las tres especies de macroalgas más comunes que varan sobre las playas arenosas de la costa de Valdivia (ca. $39^{\circ}$ $\mathrm{S})$ : Macrocystis pyrifera, Lessonia nigrescens (Bory) y Durvillaea antarctica (Chamisso) Hariot (Jaramillo et al. 2006, Duarte 2007). Ya que las macroalgas estudiadas presentan diferencias significativas entre sus contenidos de energía y constituyentes orgánicos (e.g., carbohidratos y proteínas) (Gómez \& Westermeier 1995) y a que juveniles y adultos pueden diferir en sus estrategias alimentarias (e.g., Pavia et al. 1999, Yu et al. 2003), los resultados de este estudio se discuten en relación a algunos mecanismos morfológicos y fisiológicos que podrían dar cuenta de esas diferencias. Teniendo presente que la partición espacio-temporal que muestran juveniles y adultos de $O$. tuberculata, ha sido interpretada como un medio a fin de que los primeros eviten el canibalismo que los adultos ejercen sobre ellos (Kennedy et al. 2000, Duarte 2007), se discuten además los patrones de preferencia en relación a competencia intraespecífica entre ambos estadios ontogénicos.

\section{MATERIALES Y MÉTODOS}

\section{Recolección de animales experimentales}

Los individuos adultos y juveniles de $O$. tuberculata fueron recolectados durante noviembre de 2004, desde la zona intermareal de la playa de Calfuco, localizada en la costa de 
Valdivia, centro sur de Chile (ca. 39 $\mathrm{S}$ ) (Fig. 1). Se seleccionó esta playa debido a las altas abundancias poblacionales de este anfípodo en la misma: durante los períodos enero-marzo 1995 y 1996 (E. Jaramillo, resultados no publicados), las densidades de $O$. tuberculata en esta playa alcanzaron valores promedios cercanos a 2.200-2.400 individuos $\mathrm{m}^{-2}$. La obtención de los individuos se realizó excavando con la mano en sus áreas de enterramiento y durante las horas de luz. Los anfípodos recolectados se mantuvieron con arena húmeda hasta su posterior traslado al laboratorio. Una vez en el laboratorio, los organismos se transfirieron a cajas plásticas de
$17 \times 12 \times 6 \mathrm{~cm}$, cubiertas por tapas previamente perforadas y con una capa de arena húmeda de $3 \mathrm{~cm}$ de espesor. Los juveniles fueron aquellos individuos cuyas longitudes corporales (i.e., longitud cefalotóraxica) fue $<7,0 \mathrm{~mm}$, los cuales no presentaron caracteres sexuales secundarios como gnatopodos más grandes en los machos y láminas incubatrices en las hembras, mientras que los adultos fueron aquellos individuos con longitud corporal de $>$ $14,0 \mathrm{~mm}$ y caracteres sexuales secundarios (Jaramillo et al. 1980). No se incluyeron aquellos individuos entre 7 y $14 \mathrm{~mm}$ a fin de contar con estados ontogénicos completamente diferenciados.

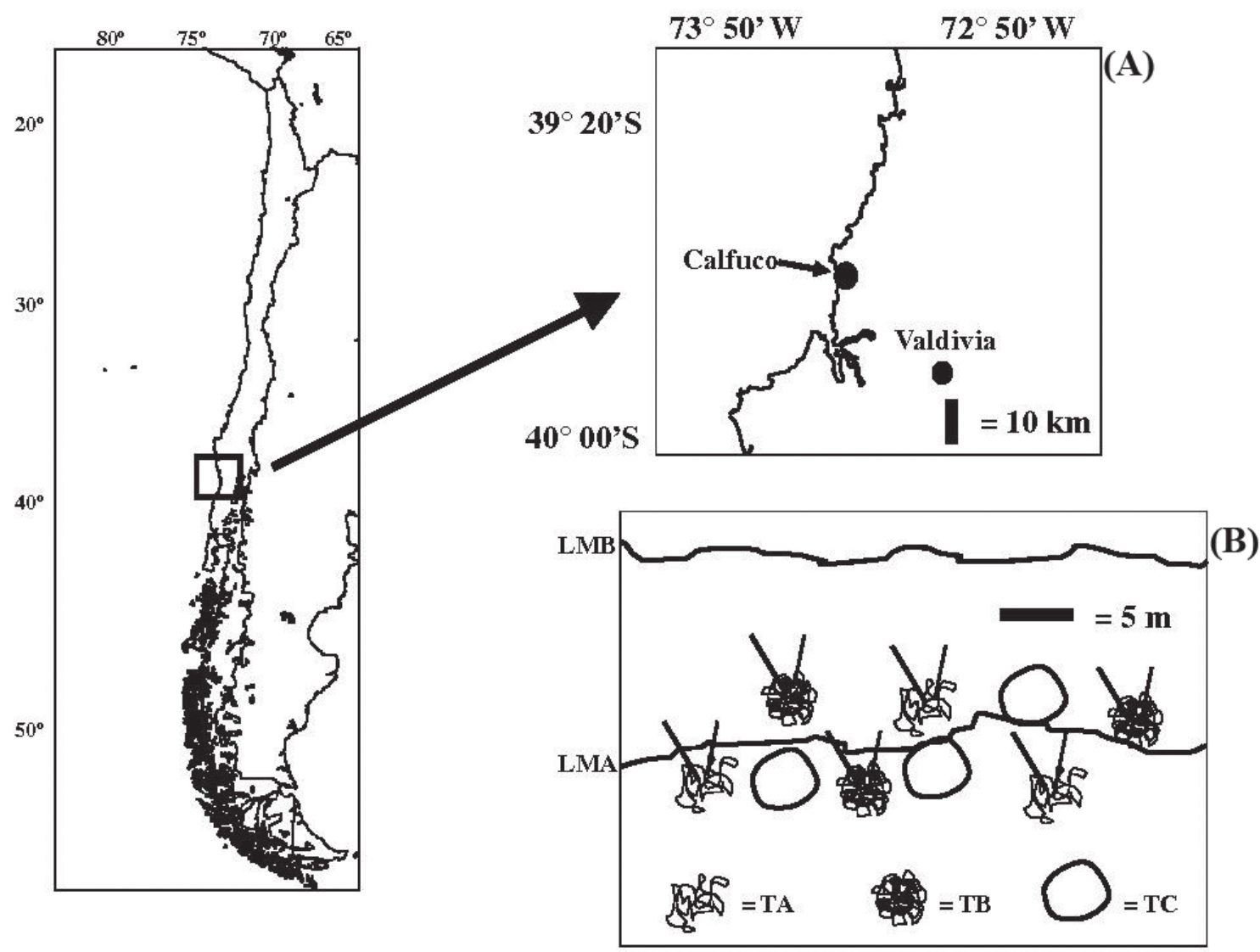

Fig. 1: (A) Ubicación de la playa arenosa de Calfuco, centro sur de Chile. (B) Diseño experimental utilizado para analizar preferencia alimentaria de $O$. tuberculata en terreno; TA $=$ cúmulos de $D$. antarctica , TB = cúmulos de $M$. pyrifera y TC = áreas sin cúmulos de macroalgas; LMB = línea de marea baja, LMA = línea de marea alta.

(A) Location of the sandy beach of Calfuco, south central Chile. (B) Experimental design used to analyze food preference of $O$. tuberculata in the field; TA $=$ deposits of $D$. antarctica, $\mathrm{TB}=$ deposits of $M$. pyrifera and $\mathrm{TC}=$ areas without deposits of macroalgae; LMB = line of low tide, LMA = line of high tide. 


\section{Preferencia alimentaria}

La preferencia alimentaria de juveniles y adultos de $O$. tuberculata se estudió de forma independiente (aunque de manera simultánea) durante la primavera de 2004. Los animales experimentales fueron mantenidos sin alimento por 24 horas antes de comenzar los experimentos. Para evaluar la preferencia de los juveniles se utilizaron grupos de 30 individuos, en tanto que para evaluar la preferencia de los adultos se utilizaron grupos de 20 individuos, sin considerar el sexo de los mismos. Experimentos pilotos mostraron que ese número de individuos fue el adecuado para evaluar el consumo de cada uno de estos grupos ontogénicos. Ambos experimentos se desarrollaron en oscuridad permanente y a una temperatura ambiental constante de $16^{\circ} \mathrm{C}$.

Se ofrecieron en forma simultánea tres especies de macroalgas en estado fresco (i.e., recién recolectadas de las rocas) a juveniles y a adultos de $O$. tuberculata: frondas de $M$. pyrifera, D. antarctica y L. nigrescens. Estos ítemes alimentarios fueron los principales restos orgánicos depositados en la zona intermareal de las playas arenosas de la costa Valdivia, durante el período mayo 2003-mayo 2004 (Duarte 2007). Cada uno de los experimentos incluyó dos tratamientos (Tabla 1A y 1B). En ambos experimentos, el tratamiento 1 , tuvo animales experimentales (juveniles o adultos), a los cuales se les ofrecieron simultáneamente frondas de las tres especies de macroalgas ( $4 \mathrm{~g}$ de cada una aproximadamente). Las frondas fueron pesadas en una balanza analítica de 0,0001 g de precisión. En ambos experimentos, el tratamiento 2 solo tuvo frondas de las tres especies de macroalgas sin animales experimentales y fue utilizado como control. Cada uno de los tratamientos en cada uno de los experimentos fue replicado seis veces. Este diseño experimental fue similar al propuesto por Roa (1992) y Manly (1993); sin embargo en este estudio, cada una de las réplicas del tratamiento 1 tuvo asociada una réplica correspondiente al tratamiento control en una arena experimental idéntica, pero separada por una hoja plástica (Silva et al. 2004). Es decir, ambas réplicas (i.e., tratamiento y control) fueron afectadas por las mismas condiciones a lo largo del experimento. El propósito de esa modificación fue evitar las numerosas posibles combinaciones de réplicas (i.e., tratamiento versus control) que resultan del diseño propuesto por Manly (1993) y que pueden llevar a numerosos resultados de difícil interpretación (Manly 1993). La distribución espacial de las réplicas en cada uno de los experimentos fue al azar. Al final del cuarto día después del inicio de los experimentos, se examinaron las cajas experimentales para evaluar el consumo de las frondas de las diferentes algas mediante cálculos de diferencias de peso.

TABLA 1

Diseño experimental para analizar las preferencias alimentarias de juveniles (A) y adultos (B) de $O$. tuberculata en condiciones de laboratorio. En cada uno de los experimentos, el tratamiento 1 incluyó los tres ítemes alimentarios (macroalgas) y anfípodos juveniles o adultos, mientras que el tratamiento 2 solo incluyó los ítemes alimentarios (i.e., sin anfípodos).

Cada tratamiento tuvo seis réplicas

Experimental design to analyze the food preferences of juveniles (A) and adults (B) of O. tuberculata in laboratory conditions. At each experiment, treatment 1 included the three food items (macroalgae) and juvenile or adult amphipods, while treatment 2 included only food items (i.e. without amphipods). Each treatment had six replicates

\begin{tabular}{|c|c|c|c|c|}
\hline Tratamientos & & Ítem alimentario & & Anfípodos \\
\hline \multicolumn{5}{|l|}{ (A) } \\
\hline 1 & D. antarctica & L. nigrescens & $M$. pyrifera & Juveniles \\
\hline 2 & D. antarctica & L. nigrescens & M. pyrifera & Ausentes \\
\hline \multicolumn{5}{|l|}{ (B) } \\
\hline & D. antarctica & L. nigrescens & $M \cdot$ pyrifera & Adultos \\
\hline & D. antarctica & L. nigrescens & M. pyrifera & Ausentes \\
\hline
\end{tabular}




\section{Experimentos de terreno}

Para evaluar experimentalmente el rol de los cúmulos de macroalgas sobre las abundancias de juveniles y adultos de $O$. tuberculata se realizaron experimentos de terreno durante noviembre de 2004 en la playa de Calfuco (Fig. 1). Esto permitió comparar los patrones de preferencias alimentarias observados en condiciones de laboratorio con las preferencias observadas en condiciones de terreno. Se recolectaron restos de macroalgas de las especies $M$. pyrifera y $D$. antarctica, en estado fresco, es decir aquellas recién depositadas sobre la zona intermareal. No se incluyó $L$. nigrescens en este experimento debido a la escasa cantidad de restos de esta macroalga, durante el día del experimento. Las frondas de $M$. pyrifera y $D$. antarctica fueron dispuestas alrededor de la línea de última marea (Fig. 1). Para ello se utilizaron varillas plásticas de $1 \mathrm{~m}$ que sirvieron de anclaje (Fig. 1). La biomasa de las macroalgas (ver abajo) fue estimada con una balanza de mano de $1 \mathrm{~g}$ de precisión y su peso fue de aproximadamente $3 \mathrm{~kg}$ en cada una de las réplicas. Previo a esto, las macroalgas fueron cuidadosamente lavadas con agua de mar, a fin de eliminar de las mismas la presencia de anfípodos.

El diseño experimental constó de tres tratamientos con tres réplicas cada uno: los tratamientos A y B incluyeron frondas frescas de las especies $D$. antarctica y $M$. pyrifera, respectivamente, en tanto que el tratamiento $\mathrm{C}$ (o control) no incluyó frondas de esas macroalgas, es decir fueron áreas de la playa sin cúmulos experimentales de algas (Fig. 1). La disposición de las réplicas de todos los tratamientos fue al azar. El experimento tuvo una duración de 12 horas (20:00-08:00 h), es decir abarcó un ciclo mareal completo. Una vez finalizado el experimento, se recolectaron muestras de sedimento desde cada una de las réplicas de los distintos tratamientos, con un cilindro plástico de $0,05 \mathrm{~m}^{2}$ de área enterrado a una profundidad de $30 \mathrm{~cm}$ en el sedimento. Por lo tanto, en los tratamientos A y B las muestras incluyeron restos de las macroalgas junto con los sedimentos incluidos bajo las mismas. Las muestras se filtraron a través de mallas con 1 $\mathrm{mm}$ de abertura; los anfípodos retenidos fueron mantenidos en formalina al $5 \%$ hasta su posterior análisis en laboratorio.

\section{Análisis de los datos}

Para evaluar la hipótesis nula de que juveniles y adultos de $O$. tuberculata no muestran preferencias por los distintos tipos de macroalgas en condiciones de laboratorio, se utilizó la prueba de Friedman de dos vías no paramétrica (Zar 1999). Se utilizó esta prueba no paramétrica debido a la falta de independencia de los tratamientos (i.e., ítemes alimentarios): el consumo de un ítem alimentario depende de la presencia de otro, y a que esa dependencia es capturada por los análisis estadísticos basados en rangos tal como la prueba de Friedman (Silva et al. 2004). Esta prueba fue desarrollada para juveniles y adultos de $O$. tuberculata, separadamente. El primer paso en el análisis fue obtener las diferencias entre el peso inicial y el peso final después de cuatro días de los ítemes alimentarios desde cada uno de los tratamientos (i.e., T1 y T2) (Tablas 2 y 3). El cambio natural (i.e., aquel que fue independiente del consumo) que registró cada ítem alimentario a través del experimento, fue eliminado substrayendo la diferencia entre el peso inicial y el peso final del ítem alimentario del control (i.e., sin consumidores) a la diferencia entre el peso inicial y el peso final del ítem alimentario de su correspondiente réplica en el tratamiento 1 (i.e., con consumidores). El siguiente paso fue calcular las diferencias entre los valores de los diferentes ítemes alimentarios. Debido a los cambios autogénicos de los ítemes alimentarios ofrecidos (los cuales fueron evaluados con el control) en algunos casos se obtuvieron valores negativos de consumo o de cambio natural o viceversa (Roa 1992, Silva et al. 2004). Sin embargo, los consumos y cambios autogénicos no deben evaluarse por réplicas sino por tratamientos, es decir por el conjunto total de datos. Finalmente, los valores promedios del nuevo set de datos fueron evaluados con la prueba de Friedman (Silva et al. 2004). Una prueba a posteriori equivalente a LSD ("least significant difference") incluida en el paquete estadístico StatsDirect, fue utilizada para evaluar cuál o cuáles ítemes alimentarios fueron preferidos.

Para comparar las abundancias de juveniles y adultos de $O$. tuberculata en los diferentes tratamientos experimentales de terreno, se utilizó análisis de varianza de una vía para cada uno de esos grupos ontogénicos (ANDEVA) 
(Zar 1999). Los datos fueron previamente transformados con $\log (x+1)$, para cumplir con los supuestos básicos de normalidad y homocedasticidad del análisis, los cuales fueron evaluados con las pruebas de KolmogorovSmirnov y Bartlett, respectivamente (Zar 1999). La prueba de Kolmogrov-Smirnov, mostró valores de significancia $>0,15$ y 0,06 para juveniles y adultos, respectivamente, en tanto que la prueba de Bartlett registró valores de probabilidad de 0,23 y 0,72 para juveniles y adultos, respectivamente. Cuando el ANDEVA mostró diferencias, se utilizó la prueba a posteriori HSD ("honestly significant difference") de Tukey para determinar cuáles promedios difirieron entre sí (Zar 1999).

TABLA 2

Resultados del consumo y cambios autogénicos en el peso de las macroalgas ofrecidas simultáneamente a juveniles de $O$. tuberculata en condiciones de laboratorio. Los valores corresponden al peso inicial menos el peso final. Se entrega el valor de $\mathrm{T}_{2}$ de la prueba de Friedman y probabilidad asociada $(\mathrm{P})$ además de los resultados de la prueba a posteriori (ver Materiales y Métodos para detalles)

Results of consumption and autogenic changes in weight of macroalgae offered simultaneously to juveniles of $O$. tuberculata in laboratory conditions. The values correspond to the initial weight minus final weight of those plants. Values

of $\mathrm{T}_{2}$ from Friedman's test and associated probability $(\mathrm{P})$, and the results of the a posteriori tests are also given (see Material and Methods for details)

\begin{tabular}{|c|c|c|c|c|c|c|}
\hline \multirow[t]{3}{*}{ Réplicas } & \multicolumn{6}{|c|}{ Macroalga } \\
\hline & \multicolumn{2}{|c|}{ D. antarctica } & \multicolumn{2}{|c|}{ L. nigrescens } & \multicolumn{2}{|c|}{ M. pyrifera } \\
\hline & Consumo (g) & Control (g) & Consumo (g) & Control (g) & Consumo (g) & Control (g) \\
\hline 1 & 0,22 & 0,16 & 0,86 & $-0,01$ & 0,05 & $-0,40$ \\
\hline 2 & 0,30 & 0,04 & 0,61 & $-0,11$ & $-0,07$ & $-0,31$ \\
\hline 3 & $-0,14$ & 0,25 & 1,11 & $-0,30$ & $-0,67$ & $-1,08$ \\
\hline 4 & $-0,02$ & 0,15 & 1,26 & $-0,05$ & $-0,34$ & $-0,14$ \\
\hline 5 & 0,18 & 0,03 & 1,18 & $-0,50$ & 0,02 & $-0,07$ \\
\hline 6 & 0,30 & 0,16 & 1,02 & $-0,03$ & $-0,06$ & $-0,05$ \\
\hline
\end{tabular}

$\mathrm{T}_{2}=15, \mathrm{P}<0,01$ L. nigrescens $>$ D. antarctica $=$ M. pyrifera

TABLA 3

Resultados del consumo y cambios autogénicos en el peso de las macroalgas ofrecidas simultáneamente a adultos de $O$. tuberculata en condiciones de laboratorio. Los valores corresponden al peso inicial menos el peso final. Se entrega el valor de $\mathrm{T}_{2}$ de la prueba de Friedman y probabilidad asociada $(\mathrm{P})$ además de los resultados de la prueba a posteriori (ver Materiales y Métodos para detalles)

Results of consumption and autogenic changes in weight of macroalgae offered simultaneously to adults of $O$. tuberculate in laboratory conditions. The values correspond to the initial weight minus final weight of those plants. Values of $\mathrm{T}_{2}$ from

Friedman's test and associated probability $(\mathrm{P})$, and the results of the a posteriori tests are also given (see Material and Methods for details)

\begin{tabular}{|c|c|c|c|c|c|c|}
\hline \multirow[t]{3}{*}{ Réplicas } & \multicolumn{6}{|c|}{ Macroalga } \\
\hline & \multicolumn{2}{|c|}{ D. antarctica } & \multicolumn{2}{|c|}{ L. nigrescens } & \multicolumn{2}{|c|}{ M. pyrifera } \\
\hline & Consumo (g) & Control (g) & Consumo (g) & Control (g) & Consumo (g) & Control (g) \\
\hline 1 & 2,82 & 0,16 & 0,33 & $-0,01$ & 0,09 & $-0,40$ \\
\hline 2 & 3,33 & 0,04 & 0,17 & $-0,11$ & $-0,14$ & $-0,31$ \\
\hline 3 & 1,69 & 0,25 & 0,32 & $-0,30$ & $-0,04$ & $-1,08$ \\
\hline 4 & 3,35 & 0,15 & 0,03 & $-0,05$ & 0,02 & $-0,14$ \\
\hline 5 & 3,46 & 0,03 & 0,40 & $-0,50$ & $-0,03$ & $-0,07$ \\
\hline 6 & 3,52 & 0,16 & 0,11 & $-0,03$ & $-0,09$ & $-0,05$ \\
\hline
\end{tabular}

$\mathrm{T}_{2}=15, \mathrm{P}<0,01$ D. antarctica $>$ L. nigrescens $=M$. pyrifera 


\section{RESULTADOS}

\section{Experimentos de preferencia en laboratorio}

Los juveniles de $O$. tuberculata, mostraron un consumo promedio diario (peso húmedo x 30 individuos), de $2,1 \mathrm{mg}(\mathrm{DE}=60,6), 293,3 \mathrm{mg}$ $(\mathrm{DE}=89,7)$ y $41,0 \mathrm{mg}(\mathrm{DE}=63,1)$, para $D$. antarctica, L. nigrescens y $M$. pyrifera, respectivamente (Fig. 2). Por otra parte, el consumo promedio diario (peso húmedo x 20 individuos), de los organismos adultos de $O$. tuberculata, fue de $724,2 \mathrm{mg}(\mathrm{DE}=191,2)$, $98,3 \mathrm{mg}(\mathrm{DE}=78,0)$, y 77,5 mg $(\mathrm{DE}=100,2)$ para $D$. antarctica, L. nigrescens y $M$. pyrifera, respectivamente (Fig. 2). El consumo total de macroalgas (i.e., independiente del tipo de alga) de los adultos fue tres veces más alto que el registrado por los juveniles.

Los resultados de la prueba de Friedman muestran que los juveniles de $O$. tuberculata presentaron diferencias significativas en los patrones de preferencia entre los distintos ítemes alimentarios ofrecidos $\left(\mathrm{T}_{2}=15, \mathrm{P}<\right.$ 0,01 ) (Tabla 2). Las comparaciones a pares realizadas posteriormente (Silva et al. 2004) mostraron que L. nigrescens fue el ítem alimentario preferido significativamente por esos individuos $(\mathrm{P}<0,01)$ (Tabla 2, Fig. 2).

Los adultos de $O$. tuberculata, también mostraron diferencias significativas en los patrones de preferencia de los ítemes alimentarios ofrecidos $(\mathrm{T} 2=15, \mathrm{P}<0,01)$ (Tabla 3). Las comparaciones a pares realizadas posteriormente (Silva et al, 2004), mostraron que $D$. antarctica fue la macroalga preferida significativamente por esos individuos (Tabla 3, Fig.2).

\section{Experimentos de terreno}

Los juveniles mostraron abundancias de 7,0 individuos $0,03 \mathrm{~m}^{-2}(\mathrm{DE}=1,0)$ y 5,0 individuos $0,03 \mathrm{~m}^{-2}(\mathrm{DE}=2,6)$ en los sedimentos subyacentes a los cúmulos experimentales de $D$. antarctica y $M$. pyrifera, respectivamente y de 12,7 ( $\mathrm{DE}=3,2)$ en los sedimentos sin esos cúmulos. Los análisis de varianza mostraron que esas abundancias no difirieron entre los distintos tratamientos experimentales $\left(\mathrm{F}_{4,10}=\right.$ 4,91; $\mathrm{P}=0,06$ ) (Fig. 3). Por otra parte, los adultos registraron abundancias de 197,7 individuos $0,03 \mathrm{~m}^{-2}(\mathrm{DE}=71,7)$ y 63,0 individuos $0,03 \mathrm{~m}^{-2}(\mathrm{DE}=14,0)$ en los sedimentos subyacentes a los cúmulos experimentales de $D$. antarctica y $M$. pyrifera, respectivamente y 15,0 individuos $0,03 \mathrm{~m}^{-2}$ (DE $=6,2$ ) en los sedimentos sin esos cúmulos. Los análisis de varianza mostraron diferencias en las abundancias de los adultos entre los distintos tratamientos $\left(\mathrm{F}_{4,10}=34,83 ; \mathrm{P}<0,01\right)$ (Fig. 3). La prueba a posteriori HSD de Tukey mostró que esas abundancias fueron significativamente más altas en los sedimentos subyacentes a los cúmulos experimentales de $D$. antarctica, que las registradas en los sedimentos bajo los cúmulos experimentales de M. pyrifera (Fig. 3). Además, las abundancias de los adultos fueron significativamente mayores en los tratamientos con macroalgas (i.e., A y B) que en el control (i.e., C). Los resultados muestran que las abundancias de los juveniles fueron bastante menores que la de los adultos, situación que puede haber estado relacionada al hecho de que muchos de estos individuos podrían haber abandonado las arenas experimentales.

\section{DISCUSIÓN}

Los resultados de los experimentos de laboratorio muestran que juveniles y adultos de Orchestoidea tuberculata tienen preferencias significativas por ítemes alimentarios diferentes. Mientras los juveniles consumieron preferentemente restos del alga parda $L$. nigrescens, los adultos prefirieron restos de la macroalga $D$. antarctica. La marcada diferencia en los patrones de preferencia de juveniles y adultos, demuestra la importancia de considerar la variabilidad intraespecífica de los organismos, en estudios de preferencias alimentarias (Sandlin \& Willig 1993, Poore 1994, Pavia et al. 1999). Aun cuando existe una variedad de trabajos que muestran que varios anfípodos revelan preferencias por distintos ítemes alimentarios (Karez et al. 2000, Pennings et al. 2000, Adin \& Riera 2003), pocos estudios se han realizado para examinar eventuales diferencias intraespecíficas en la selección de la dieta (Salema 1987, Byrén et al. 2006). Similar a los resultados de este trabajo, Pavia et al. (1999), mostraron que mientras los adultos del anfípodo Gammarus locusta (Linnaeus, 1758) prefirieron los macroepífitos del alga parda Ascophyllum 
nodosum (Linnaeus) Le Jolis, los juveniles también se alimentaron de las partes meristemáticas de esa macroalga. A su vez, Byrén et al. (2006), encontraron que los juveniles del anfípodo Pontoporeia femorata (Kröyer) difirieron significativamente en los patrones alimentarios con sus adultos conespecíficos: mientras los juveniles consumen más fitodetritus fresco, los adultos consumen material más antiguo. Debido a que la palatabilidad de las macroalgas puede cambiar dependiendo del tiempo transcurrido desde el desprendimiento de las mismas (Rodríguez 1999, Pennings et al. 2000), este aspecto debería ser considerado en estudios futuros, a fin de ganar mayor conocimiento sobre los patrones alimentarios de este especie. Por ejemplo Rothäusler \& Thiel (2006), encontraron que $L$. nigrescens se volvió más palatable para el anfípodo Parhyalella ruffoi (Lazo \& Wamble 2001) 12 días después de haber sido desprendida del sustrato.

\section{Juveniles}

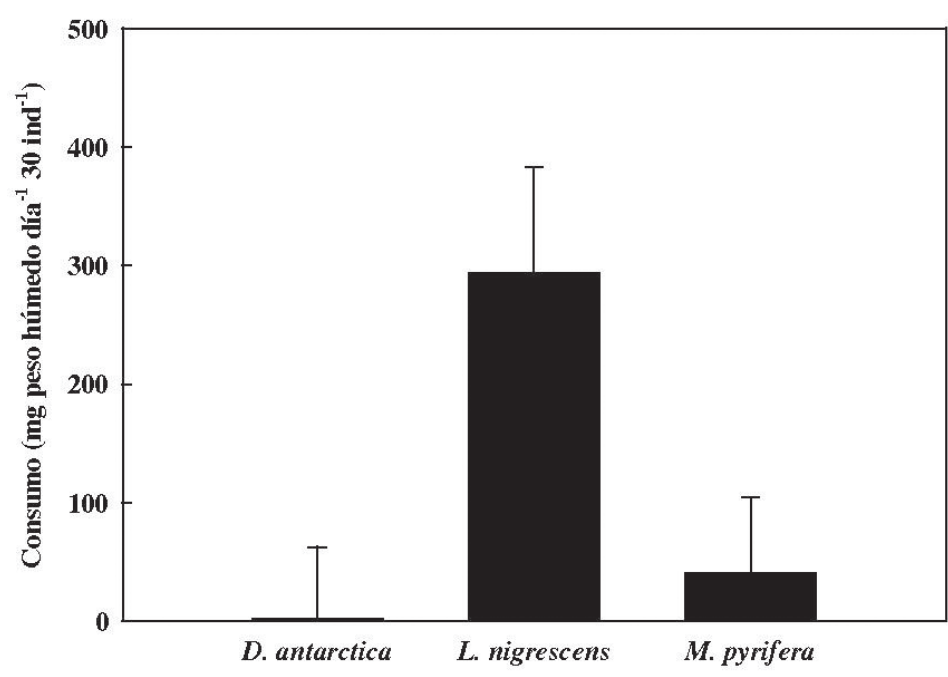

Adultos

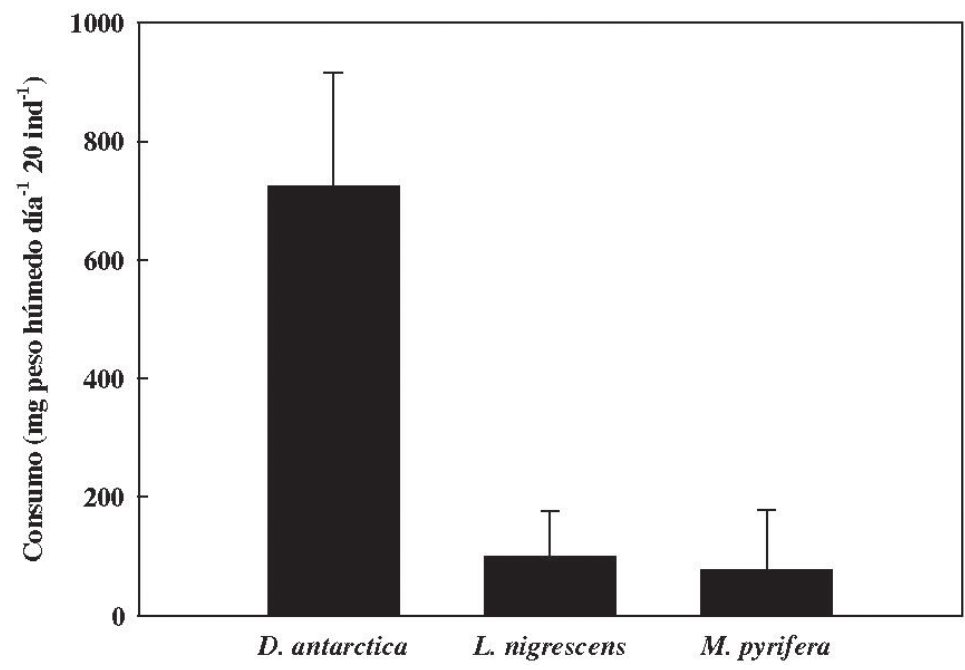

Fig. 2: Consumo promedio $( \pm 1 \mathrm{DE})$ de los ítemes alimentarios ofrecidos en condiciones de laboratorio a juveniles y adultos de $O$. tuberculata.

Mean consumption $( \pm 1 \mathrm{SD}$ ) of the food items offered in laboratory conditions to juveniles and adults of O. tuberculata. 

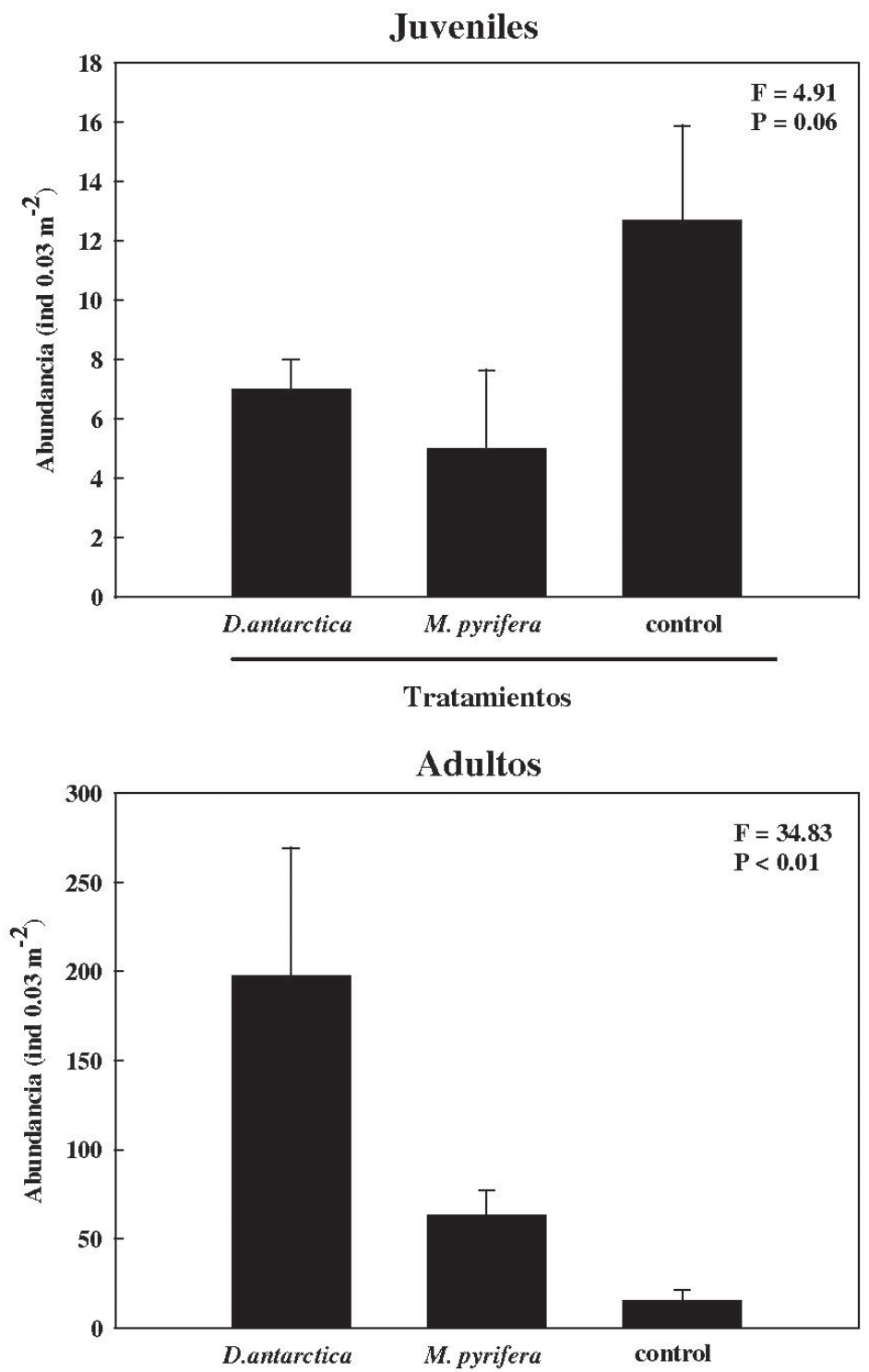

Tratamientos

Fig. 3: Abundancias promedios $( \pm 1 \mathrm{DE})$ de juveniles y adultos de $O$. tuberculata bajo depósitos de $D$. antarctica y $M$. pyrifera y en áreas sin depósitos de macroalgas. Se entregan los valores de $\mathrm{F}$ y $\mathrm{P}$ resultantes de ANDEVA para cada comparación. Las líneas unen promedios que no difieren significativamente entre sí $(\mathrm{P}>0,05)$.

Mean abundances $( \pm 1 \mathrm{SD})$ of juveniles and adults of $O$. tuberculata collected under deposits of $D$. antarctica and $M$. pyrifera and in areas without deposits of macroalgae. Values of $\mathrm{F}$ and $\mathrm{P}$ resulting from ANOVA are given for each comparison. Lines link means not significantly different among each others $(P>0.05)$.

Conocer los factores que determinan las preferencias alimentarias de los consumidores herbívoros, es uno de los aspectos más complejos en este tipo de estudios (e.g., Pennings \& Paul 1992, Penning et al. 1998). Aspectos tales como la amplia variedad de rasgos (e.g., características nutricionales y estructurales) que presentan las macrófitas (Steneck \& Watling 1982, Pennings et al. 1998, McClintock \& Baker 2001), o las diferencias inter e intraespecíficas de los requerimientos nutricionales de los herbívoros 
(Pennings et al. 2000, Byrén et al. 2006) dificultan la interpretación de los resultados. Por ejemplo, Gómez \& Westermeier (1995) estudiaron los contenidos energéticos y constituyentes orgánicos (proteínas, lípidos, carbohidratos y manitol) en las mismas especies de macroalgas utilizadas en este estudio y encontraron que L. nigrescens tiene en general un valor nutritivo más bajo que el de las otras especies, con contenidos de proteínas, carbohidratos solubles y manitol significativamente más bajos que los de $M$. pyrifera y con valores de energía total y carbohidratos solubles y manitol significativamente más bajos que los de $D$. antarctica. El hecho de que los juveniles de $O$. tuberculata tengan un consumo preferencial de L. nigrescens por sobre las otras dos macrófitas, confirma la dificultad de entender y predecir los patrones de preferencia en estos consumidores marinos (Pennings et al. 1998). Los factores que determinan el cambio ontogénico en el uso del alimento de $O$. tuberculata no son conocidos; sin embargo se proponen aquí algunas posibilidades. Primero, el cambio en los ítemes alimentarios consumidos puede estar relacionado con cambios ontogénicos en la morfología de las estructuras asociadas con la alimentación (apéndices bucales o gnatopodos). Es posible que esas diferencias influyan en la capacidad de procesar el alimento (e.g., capacidad de desgarro) de juveniles y adultos y por lo tanto sobre sus preferencias alimentarias. Similares conclusiones proponen $\mathrm{Yu}$ et al. (2003), los cuales sugieren que las diferencias en los patrones alimentarios de juveniles y adultos del anfípodo Synchelidium lenorostralum (Hirayama, 1986), podrían estar relacionados con diferencias en la morfología de las partes de la boca. De este modo, los juveniles estarían limitados a consumir solo presas de menor tamaño. Otra posibilidad plausible para explicar las diferencias en las preferencias alimentarias que muestran los estados ontogénicos de $O$. tuberculata, son las diferencias en los requerimientos nutricionales de juveniles y adultos (Byrén et al. 2006). Al menos dos aspectos diferencian a esos estados y que podrían influir en sus requerimientos energéticos y nutricionales (Abrahams \& Dill 1989). En primer lugar, la tasa de crecimiento de los juveniles es más alta que la de los adultos (Contreras et al. 2003), lo que sugiere diferentes necesidades nutricionales entre ambos estados. En segundo lugar, puede mencionarse el desarrollo gonadal que ocurre solo en individuos adultos. Por ejemplo, Jormalainen et al. (2001) sugieren que tanto las diferencias en desarrollo gonadal y tasas de crecimiento entre machos y hembras del isópodo Idotea baltica (Pallas), podrían explicar en parte, las diferencias que muestran esos estados en el consumo del alga parda Fucus vesiculosus Linnaeus en la costa sur de Finlandia. Mientras los machos prefirieron y se desarrollaron mejor consumiendo los brotes nuevos de la zona apical del alga, las hembras se desarrollaron de forma similar consumiendo brotes, tanto de la zona apical como de la zona basal de la misma, e incluso prefirieron esta última zona del alga, a pesar de la mejor calidad nutricional de la zona apical (Jormalainen et al. 2001). De este modo, los diferentes requerimientos nutricionales y de energía necesarios para crecimiento y desarrollo gonadal, podrían explicar las diferencias en los patrones de preferencia de juveniles y adultos de $O$. tuberculata. Sin embargo, es posible también que las demandas fisiológicas por sí solas no puedan explicar las preferencias alimentarias encontradas en los experimentos de laboratorio realizados en este estudio.

Aun cuando no se pudo incluir L. nigrescens en los experimentos de terreno, estos entregaron importantes antecedentes para contrastar con los resultados de los experimentos realizados en condiciones de laboratorio. Similar a los resultados de estos últimos, los juveniles de $O$. tuberculata no mostraron preferencias significativas al ofrecerles $D$. antartica y $M$. pyrifera en condiciones de terreno, a la vez que los adultos prefirieron significativamente a $D$. antarctica por sobre M. pyrifera. Estos resultados sugieren que para los adultos, D. antárctica sería importante como fuente de alimento y también de hábitat. Por otro lado, la eventual preferencia de los juveniles por L. nigrescens en terreno permanece como una pregunta abierta. Estudios utilizando estructuras inorgánicas, que simulen las condiciones bajo los cúmulos de macroalgas, permitirán obtener mayor conocimiento de si estas macroalgas 
sirven como fuente de alimento o refugio o ambas. El estudio de la distribución natural de juveniles y adultos de $O$. tuberculata en relación a la distribución de los cúmulos de macroalgas, puede ser una vía útil para evaluar y contrastar los resultados de los experimentos de preferencia alimentaria en laboratorio.

Las diferentes preferencias alimentarias observadas entre juveniles y adultos de $O$. tuberculata pueden también estar relacionadas a interacciones competitivas. Algunos autores han demostrado la existencia de depredación de adultos sobre juveniles en $O$. tuberculata (Kennedy et al. 2000, Duarte 2007). Se ha sugerido que la separación en el tiempo de la actividad locomotriz, es el principal mecanismo para reducir el contacto entre los individuos de estos dos grupos ontogénicos, maximizando de este modo la sobrevivencia de los juveniles, ya que la actividad de los adultos ocurre primariamente durante la noche mientras que la de los juveniles se concentra durante el día (Jaramillo et al. 1980, 2003, Kennedy et al. 2000). Sin embargo, la actividad de los juveniles y adultos coinciden durante el atardecer y amanecer. La preferencia de los juveniles de esta especie por $L$. nigrescens, podría ser entonces otro modo de evitar encuentros con los adultos en las horas en que ambos estados coinciden sobre la superficie de la playa.

Finalmente, los resultados de este estudio entregan información útil sobre los patrones de preferencia alimentaria de $O$. tuberculata, destacando la importancia de considerar variaciones intraespecíficas en este tipo de estudios. Se necesitan aún muchos más estudios para conocer los mecanismos últimos que determinan los patrones de alimentación en esta especie y otros invertebrados que habitan en playas arenosas.

\section{AGRADECIMIENTOS}

Este estudio fue financiado por CONICYT (beca doctoral a CD) y Dirección de Investigación y Desarrollo de la Universidad Austral de Chile (Proyecto D-2003-15 concedido a $\mathrm{CD}$ ). Los autores agradecen el apoyo en terreno de Erwin Barría y Luis Figueroa y de Marcia González, Karin Acuña y Sandra Cifuentes en labores de laboratorio.

\section{LITERATURA CITADA}

ABRAHAMS MV \& LM DILL (1989) A determination of the energetic equivalence of the risk of predation. Ecology 70: 999-1007.

ADIN R \& P RIERA (2003) Preferential food source utilization among stranded macroalgae by Talitrus saltator (Amphipod, Talitridae): a stable isotopes study in the northern coast of Brittany (France). Estuarine, Coastal and Shelf Science 56: 91-98.

BEDNEKOFF PA (1996) Risk-sensitive foraging, fitness, and life histories: where does reproduction fit into the big picture? American Zoologist 36: 471-483.

BYRÉN L, G EJDUNG \& R ELMGREN (2006) Uptake of sedimentary organic matter by the deposit-feeding Baltic amphipods Monoporeia affinis and Pontoporeia femorata. Marine Ecology Progress Series 313: 135-149.

CONTRERAS H, E JARAMILLO, C DUARTE \& A McLACHLAN (2003) Population abundances, growth and natural mortality of the crustacean macroinfauna at two sandy beach morphodynamics types in southern Chile. Revista Chilena de Historia Natural, 76: 543-561.

DAYTON PK, V CURRIE, T GERRODETTE \& BD KELLER (1984) Patch dynamics and stability of some California kelp communities. Ecological Monographs 54: 253-289.

DAYTON PK, MJ TEGNER, PE PARNELL \& PB EDWARDS (1992) Temporal and espacial patterns of disturbance and recovery in a kelp forest community. Ecological Monographs 62: 421-445.

DUARTE W (1974) Orchestoidea tuberculata Nicolet, 1894 como organismo desintegrador de algas (Crustacea, Amphipoda, Talitridae). Noticiario Mensual del Museo Nacional de Historia Natural (Chile) 220-221: 3-9.

DUARTE C (2007) Subsidios tróficos: importancia de las macroalgas varadas para el anfípodo Orchestoidea tuberculata (Nicolet), en playas de arena del centro sur de Chile. Tesis doctoral, Facultad de Ciencias, Universidad Austral de Chile, Valdivia, Chile. 140 pp.

DUFFY JE \& ME HAY (1991) Food and shelter as determinants of food choice by an herbivorous marine amphipod. Ecology 72: 1286-1298.

DUGAN J, DM HUBBARD, MD MCCRARY \& MO PIERSON (2003) The response of macrofauna communities and shorebirds to macrophyte wrack subsidies on exposed sandy beach of southern California. Estuarine Coastal and Shelf Science 58: 25-40.

GÓMEZ I \& R WESTERMEIER (1995) Energy contents and organic constituents in Antarctic south Chilean marine brown algae. Polar Biology 15: 597-602.

GRIFFITHS CL \& J STENTON-DOZEY (1981) The fauna and rate of degradation of stranded kelp. Estuarine Coastal and Shelf Science 12: 645-653.

GRIFFITHS CL, J STENTON-DOZEY \& K KOOP (1983) Kelp wrack and the flow of energy through a sandy beach ecosystem. En: McLachlan A \& T Erasmus (eds) Sandy beaches as ecosystems: 547-556. Dr. W. Junk Publishers, The Hague, The Netherlands.

INGLIS G (1989) The colonisation and degradation of stranded Macrocystis pyrifera (Linnaeus) C. Agardh, 1820. Ag. by the macrofauna of a New Zealand sandy beach. Journal of Experimental Marine Biology and Ecology 125: 203-217.

JARAMILLO E, W STOTZ, C BERTRÁN, J NAVARRO, C ROMAN \& C VARELA (1980) Actividad locomotriz de Orchestoidea tuberculata (Amphipoda, Talitridae) 
sobre la superficie de una playa arenosa del sur de Chile (Mehuín, Valdivia). Studies of Neotropical Fauna and Environment 15: 9-33.

JARAMILLO E, H CONTRERAS, C DUARTE \& MH AVELLANAL (2003) Locomotor activity and zonation of upper shore arthropods in a sandy beach of north central Chile. Estuarine Coastal and Shelf Science 58: 177-197.

JARAMILLO E, R DE LA HUZ, C DUARTE \& H CONTRERAS (2006) Algal wrack deposits and macroinfaunal arthropods on sandy beaches of the Chilean coast. Revista Chilena de Historia Natural 79: 337-351.

JORMALAINEN V, T HONKANEN, A MÄKINEN, A HEMMI \& O VESAKOSKI (2001) Why does herbivore sex matter? Sexual differences in utilization of Fucus vesiculosus by the isopod Idotea baltica. Oikos 93: 77-86.

KAREZ R, S ENGELBERT \& U SOMMER (2000) Coconsumption and protective coating: two new proposed effects of epiphytes on their macroalgal hosts in mesograzer-epiphyte-host interactions. Marine Ecology Progress Series 205: 85-93.

KENNEDY F, E NAYLOR \& E JARAMILLO (2000) Ontogenetic differences in the circadian locomotor activity rhythm of the talitrid amphipod crustacean Orchestoidea tuberculata. Marine biology 137: 511-517.

MANLY F (1993) Comments on design and analysis of multiple-choice feeding-preference experiments. Oecologia 93: 149-152.

McLACHLAN A, T WOOLDRIDGE \& AH DYE (1981) The ecology of sandy beaches in south Africa. South African Journal of Zoology 16: 219-231.

McCLINTOCK JB \& BJ BAKER (2001) Marine chemical ecology. CRC Press, Boca Raton, Florida, USA. $610 \mathrm{pp}$.

MUIR DG (1977) The biology of Talorchestia capensis (Amphipoda; Talitridae), including a population energy budget. M.Sc. thesis, University of Cape Town, Rondebosch, South Africa. 94 pp.

PAVIA H, G CERVIN, A LINDGREN \& P ABERG (1997) Effects UV-B radiation and simulated herbivory on phlorotannins in the brown alga Ascophyllum nodosum (Linnaeus). Marine Ecology Progress Series 157: 139-146.

PAVIA H, H CARR \& P ABERG (1999) Habitat and feeding preferences of crustacean mesoherbivores inhabiting the brown seaweed Ascophyllum nodosum (Linnaeus) Le Jolis. and its epiphytic macroalgae. Journal of Experimental Marine Biology and Ecology 236: 15-32.

PENNINGS SC \& VJ PAUL (1992) Effect of plant toughness, calcification, and chemistry on herbivory by Dolabella auricularia. Ecology 73: 1606-1619.
PENNINGS S, TH CAREFOOT, EL SISKA, ME CHASE \& TA PAGE (1998) Feeding preferences of a generalist salt-marsh crab: relative importance of multiple plant-traits. Ecology 79: 1968-1979.

PENNINGS S, TH CAREFOOT, M ZIMMER, JP DANKO \& A ZIEGIER (2000) Feeding preferences of supralittoral isopods and amphipods. Canadian Journal of Zoology 78: 1918-1929.

POORE, AGB (1994) Selective herbivory by amphipods inhabiting the brown alga Zonaria angustata. Marine Ecology Progress Series 107: 113-123.

ROA R (1992) Design and analysis of multiple-choice feeding-preference experiments. Oecologia 89: 509515 .

RODRÍGUEZ SR (1999) Subsidios tróficos en ambientes marinos: la importancia de las macroalgas pardas a la deriva como fuente exógena de recursos alimentarios para el erizo Tetrapigus niger (Echinodermata: Echinoidea) en el intermareal rocoso de la costa de Chile central. Tesis doctoral, Departamento de Ecología, Pontificia Universidad Católica de Chile, Chile. 172 pp.

ROTHÄUSLER E \& M THIEL (2006) Effects of detachment on the palatability of two kelp species. Journal of Applied Phycology 18: 423-435

SALEMAA H (1987) Herbivory and microhabitat preferences of Idotea spp. (Isopoda) in the northern Baltic Sea. Ophelia 27: 1-15.

SANDLIN EA \& MR WILLIG (1993) Effects of age, sex, prior experience, and intraespecific food variation on diet composition of a tropical folivore (Phasmatodea: Phasmatidae). Environmental Entomology 22: 625-633.

SCAPINI F, L CHELAZZI, I COLOMBINI \& M FALLACI (1992) Surface activity, zonation and migrations of Talitrus saltator on a Mediterranean beach. Marine Biology 112: 573-581.

SILVA J, A LARRAÍN, E BAY-SCHMITH \& R. ROA (2004) Feeding-regime experiments to enhance gamete production in the carnivorous sea urchin Arbacia spatuligera. Aquaculture 231: 279-291.

STENECK RS \& L WATLING (1982) Feeding capabilities and limitation of herbivorous molluscs: a functional approach. Marine Biology 68: 299-312.

YU OK, HL SUH \& Y SHIRAYAMA (2003) Feeding ecology of the three amphipod species Synchelidium lenorostralum, S. trioostegitum and Gitanopsis japonica in the surf zone of a sandy shore. Marine Ecology Progress Series 258: 189-199.

ZAR JH (1999) Biostatistical analysis. Fourth edition. Prentice-Hall, Upper saddle River, New Jersey, USA: $662 \mathrm{pp}$.

ZIMMER M (1999) The fate and effects of ingested hydrolyzable tannins in Porcellio scaber. Journal of Chemical Ecology 25: 611-628. 
